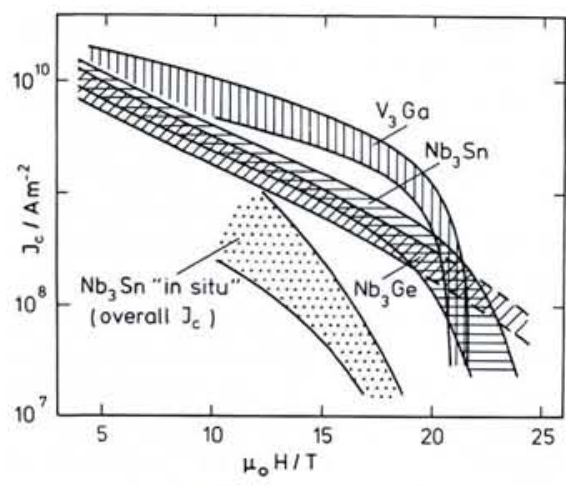

Fig. 3 - Typical short sample critical current densities vs. transverse magnetic field at $4.2 \mathrm{~K}$

Superconductivity: Tool in Metallurgy

The work with superconducting alloys is not just a race towards better conductors. When investigating new alloy systems, metallurgists make use of a number of physical techniques, and it is precisely because superconductivity is so wide-spread in metallic materials that its observation is so useful in ascribing the correct compositiontemperature coordinates of certain phase fields. We thus have an additional analytical tool of extremely good sensitivity.

The interesting point is that the sample quantity needed to detect a well defined diamagnetic transition is incredibly small. The absolute record in this respect belongs to the SQUID magnetometer - a device kindly provided by superconducting flux quantization. With this, it is almost always possible to pick out from a polycrystalline specimen, for isolated investigation, a barely visible single crystal. Another attractive possibility of observing the second order transition at $T_{c}$ - even in poor quality samples - is the measurement of the specific heat. In this case, the analysis of the anomalous electronic part yields information on possible inhomogeneous phase distributions or concentration fluctuations. Fortunately, again, modern low temperature calorimetric techniques allow the use of very small specimens, in favourable cases, a mass of the order of $1 \mathrm{mg}$ is enough. Of course, in many routine investigations, such elaborate experimental methods are not necessary, and quite often, a simple permeability-temperature scan on powdered specimens may suffice. It is instructive to note that in the past, when measurements made on superconducting transitions as a function of alloy composition appeared to be inconsistent with some well established rules, the explanation was always simple: the previously constructed phase diagram was wrong.

Superconducting technology, because of its unique possibilities, is expected to grow steadily in importance. A great deal of metallurgy will be needed to cope with major problems. From a somewhat different view-point, superconductivity still assumes another function: that of a motor in materials science.

\title{
Metallic Glasses
}

\author{
R.M.J. Cotterill, Lyngby \\ The Technical University of Denmark
}

Covalently-bonded glasses, such as the common silicate varieties, have been in use for at least 4000 years. Glass beads, dating from about $2500 \mathrm{BC}$, have been found in Egypt and other parts of the Near East, although exploitation of the plasticity of hot glass is more recent. These materials have so dominated the scene that the existence of the glassy state was believed to be intimately connected with the presence of covalent bonds. Glass has long been known to be a supercooled liquid, and covalent bonding was regarded as a pre-requisite if crystallization was to be avoided. This attitude has recently undergone radical revision. With the production of metallic examples, it has suddenly transpired that a glass need not be transparent, brittle or insulating. Glass possesses a unique lack of order, and it is this atomic-level attribute which is now acknowledged as the defining characteristic of this type of material.

It is not often that branches of science and technology as venerable as metallurgy and glass fabrication witness a genuine revolution. When both these fields simultaneously experienced such an upheaval, in 1960 , it was not surprising that the repercussions were widespread. In producing the first appreciable amounts of metallic glass, Pol Duwez, William Klement, and Ronald Willens effectively rewrote the definitions of both classes of material. Until their breakthrough, the distinction between these forms of matter had been reflected in a marked difference in the chemical compositions. The workhorses of metallurgical practice are iron, copper, zinc, aluminum, lead, nickel, and cobalt. The familiar glasses, on the other hand, are formed from compounds, in which silicon, oxygen, and sodium are particularly prominent.

The free energy barrier separating the non-crystalline and crystalline states of metals is rather small, and slow cooling of a metallic melt does not yield a glass. It has long been known however, that metals are capable of exhibiting properties other than those of their natural state. The simple trick is to cool them so rapidly that a nonequilibrium state is frozen in, this process being known as quenching. A cooling rate of tens of millions of degrees per second is required to trap a metal in its amorphous state, and this can be achieved in a variety of ways. The original method involved propelling molten globules against a cold flat surface, a process known as splat quenching. More recent methods include pouring the liquid between rapidly counterrotating drums and squirting a thin jet of the liquid against the cooled surface of a revolving cylinder. The last of these produces a continuous ribbon, at rates of up to $2000 \mathrm{~m} / \mathrm{min}$ and probably holds the greatest promise for commercial exploitation. The quite different technique of vapour condensation is also effective, and there were reports in the 1930s, notably by J. Brill and J. Kramer, of metal glass formation by this method. Credit for the revolution must also be accorded to these pioneers.

Congealed metallic melts are frequently referred to as amorphous alloys, emphasizing the fact that they are mixtures rather than pure metals. They have invariably belonged to one of two groups: a transition, or a noble metal with a smaller metalloid, or a mixture of two transition metals. Examples of the first type are $\mathrm{Au}_{75} \mathrm{Si}_{25}$ (the first combination studied by Duwez, Klement, and Willens), $\mathrm{Pd}_{80} \mathrm{Si}_{20}$ and $\mathrm{Fe}_{80} \mathrm{~B}_{20}$. The other group includes $\mathrm{Ni}_{60} \mathrm{Nb}_{40}$ and $\mathrm{Cu}_{66} \mathrm{Zr}_{34}$. Many of the more recently developed metallic glasses have more complicated compositions, such as $\mathrm{Fe}_{75} \mathrm{P}_{16} \mathrm{~B}_{6} \mathrm{Al}_{3}$ and $\mathrm{Pd}_{16} \mathrm{Au}_{66} \mathrm{Si}_{18}$. Production cost is relatively low because the glassy alloys are formed directly from the molten metal, so it is possible to bypass expensive and energy-consuming stages such as casting, rolling, and drawing, which have been necessary when working with the crystalline state of the material. One technological limitation arises from the fact that a metallic glass reverts to the crystalline form if the temperature is raised to roughly half the melting point on the absolute Kelvin scale.

One of the best known models of the liquid state is that usually accredited to John Bernal, although variants of the same approach had been discussed by Peter Debye and $\mathrm{H}$. Menke, and also by Joel Hildebrand and W.E. Morrell, both in the 1930s. Bernal's version, dating from 1959, involved pouring spheres into an irregularly-walled container to produce what is known as random close packing. As John Finney subsequently demonstrated, the model is actually more applicable to metallic glasses, particularly when the structure is produced with two different sizes of sphere, with appropriate diameters. It must be emphasized that such models do not exhibit the total randomness of an ideal gas, because the dense packing imposes quite severe geometrical constraints. A certain degree of short-range order exists, even though longrange order is completely lacking. This has led some to conclude that metallic glasses are actually polycrystals with a grain size of near atomic dimensions. Indeed, it has 


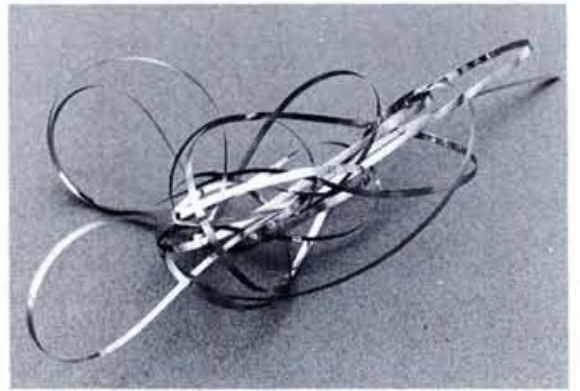

Fig. 1 - Metallic glass ribbon $\left(\mathrm{Fe}_{78} \mathrm{Mo}_{2} \mathrm{~B}_{20}\right.$ ) produced by the rapid cooling of a molten jet against a rotating drum. (Material supplied by Allied Chemical Corp., Morristown, New Jersey.)

been suggested that a glass might be essentially a crystal that is saturated with dislocations, but it is doubtful that one can identify such line defects when there is no regular lattice left to act as a reference structure. The dislocation concentration would have to be so high that the concept might lose its normal significance.

A more promising approach was put forward by Colin Goodman, and independently by Rong Wang and M.D. Merz, in 1975. Noting that systems which form glasses have as a major constituent a material that exists in several polymorphic crystalline forms, differing only slightly in free energy, they suggested that polymorphism might actually be a prerequisite for glass formation. With roughly the same free energy, and therefore approximately the same probability of occurrence, the various polymorphs would vie with one another as the liquid cools towards the glass temperature. Because of the competition, none would dominate, and the result would be a mixture of clusters of different polymorphs. There would inevitably be mismatch at the cluster interfaces. Initially, the assembly of clusters would be in a dynamic state, individual atoms constantly being shuffled from one polymorphic cluster to another. At the glass temperature, the situation would become static, with the clusters firmly bonded at their contact surfaces.

The random close-packed structure has won virtually universal acceptance as a reliable, though approximate, model of a metallic glass. When presenting it, Bernal emphasized the primitive state of our understanding of random geometry, and he suggested that the structure should be analysed in terms of the shapes and sizes of the interstitial holes it contains. Somewhat suprisingly, rather few different types of holes account for nearly all the space between the atoms. The most common type is the tetrahedron, about $85 \%$ of all holes having this form, while no other type accounts for more than $6 \%$. The other common types are the octahedron, the trigonal prism, the archimedean square antiprism, and the tetragonal dodecahedron. In 1972, D.E. Polk suggested that the spheres in a random close-packed assembly can be compared with the larger atomic species in a glassy alloy, and that the smaller atoms fit into the largest holes in the structure. The tetrahedral hole is too small to be useful in this respect, but the octahedral and trigonal prismatic holes appear to be viable candidates, especially if the smaller atoms are ionized. It was subsequently found that the metalloid elements are indeed present as ions when they are constituents of metallic glasses.

Many glassy metals are remarkably resistant to corrosion. Glassy $\mathrm{Fe}_{72} \mathrm{Cr}_{8} \mathrm{P}_{13} \mathrm{C}_{7}$, in a $10 \%$ solution of $\mathrm{FeCl}_{3}$ at $60^{\circ} \mathrm{C}$, corrodes at a rate that is less than 100 nanometres per year! Stainless steel, under the same conditions, is eaten away at about a million times this rate. This impressive disparity might imply the absence of well defined crystal boundaries and other defects in metallic glasses as in crystalline materials, defects are notoriously vulnerable to attack by corrosive agents, being slightly more open regions of the structure and thus susceptible to penetration. The lack of such inhomogeneities in these glasses would be a strong factor in reducing their tendency to oxidize. If this interpretation is valid, it is evidence against the microcrystal and dislocation models of the amorphous state.

Much of the interest in metallic glasses can be traced to a prediction, by I.A. Gubanov, that some of them would display ferromagnetism. In view of the interatomic coupling effects underlying the ferromagnetic condition, and the reinforcing factor arising from crystal symmetry, Gubanov's conjecture sounded improbable. Ferromagnetic glasses were nevertheless discovered, and hold great technological promise, particularly for use in transformers. Energy dissipation in traditional polycrystalline transformer cores represents a loss which, in 1980 , was estimated to amount to $\$ 200 \mathrm{M}$ in the USA alone.

Glassy $\mathrm{Fe}_{86} \mathrm{~B}_{7} \mathrm{C}_{7}$ has a saturation magnetization which is almost as high as the best crystalline examples, but with the advantage of a low coercivity; it is easily magnetized and demagnetized, and the hysteresis losses are thus greatly reduced. It is believed that this behaviour too is a result of the absence of grain boundaries. These and other defects are known to constitute barriers to the motion of magnetic domain walls, and their absence probably produces magnetic softness. This property also suggests applications to magnetic memory devices, like those in computers and tape recording heads, because it would facilitate rapid recording and erasure of information. Unfortunately metallic glasses fall somewhat short of the ideal because inhomogeneities in the form of slight variations of composition are difficult to eliminate completely. Some ferromagnetic glasses have extremely high permeability, making them potentially useful for miniature microphones and transformers. Others display unusually low magnetostriction, that is change in geometrical dimensions when placed in a magnetic field, which suggests use in transducers. Similarly, their low attenuation of sound and ultrasonic waves might lead to use in novel acoustic devices such as low-loss delay lines in microwave units.

One of the most important and remarkable properties of metallic glasses is their unusual combination of strength and plasticity. The ultimate tensile strengths of some examples exceed three times that of stainless steel. Covalent glass, such as the common window variety, even when in the favourable form of thin filaments, cannot be strained in excess of $1 \%$. A metallic

Fig. 2 - Feld-ion microscopy reveals a fundamental difference between two states of condensed matter. The tungsten crystal shown above left is characterized by the regular pattern of its atomic arrangement, while the $P d_{80} S_{i}$ glass lacks long-range order. The lower pictures shown the corresponding close-packed sphere assemblies that are frequently used to model the two states.
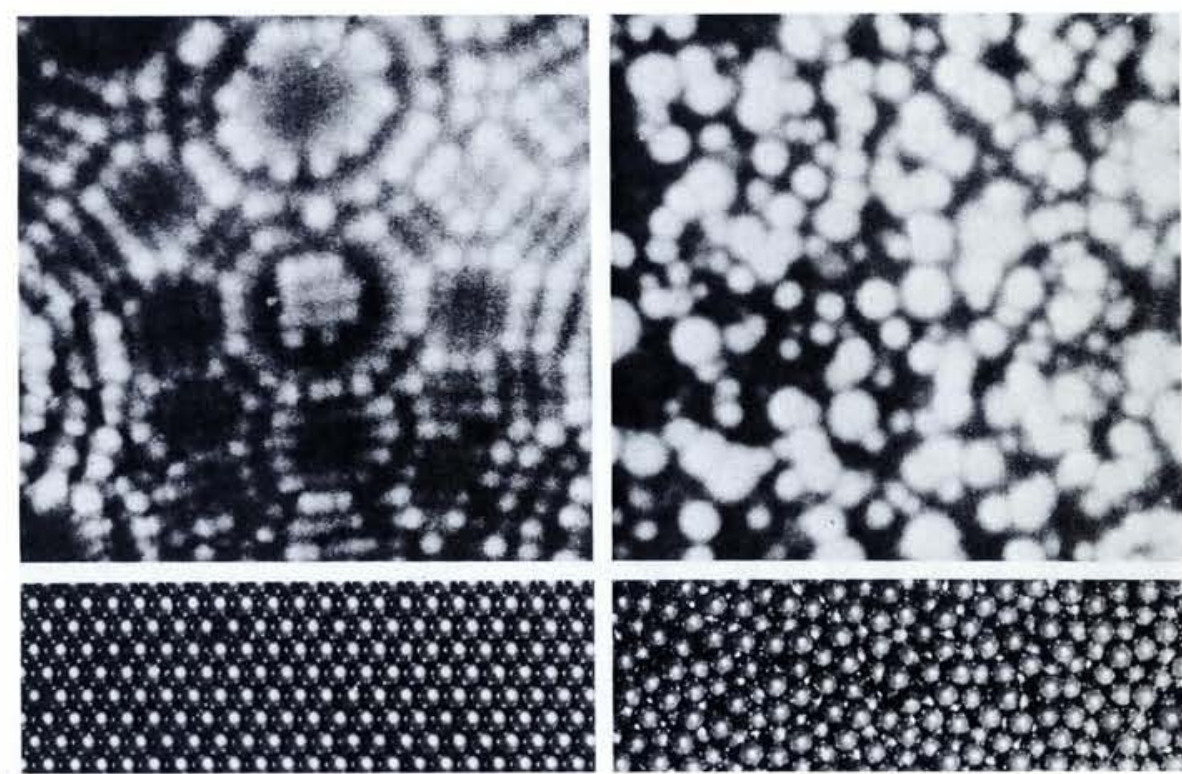


\begin{tabular}{lc}
\hline Material & $\begin{array}{c}\text { Ultimate Tensile Strength } \\
\left(\mathrm{MN} / \mathrm{m}^{2}\right)\end{array}$ \\
\hline Silicate glass fibre & 1400 \\
Crystalline steel wire & 2000 \\
$\mathrm{Pd}_{80} \mathrm{Si}_{20}$ glass & 1360 \\
$\mathrm{Cu}_{60} \mathrm{Zr}_{40}$ glass & 2000 \\
$\mathrm{Ni}_{75} \mathrm{Si}_{8} \mathrm{~B}_{17}$ glass & 2700 \\
$\mathrm{Co}_{75} \mathrm{Si}_{15} \mathrm{~B}_{10}$ glass & 3000 \\
$\mathrm{Fe}_{80} \mathrm{~B}_{20}$ glass & 3200 \\
$\mathrm{Fe}_{78} \mathrm{Si}_{10} \mathrm{~B}_{12}$ glass & 3400 \\
\hline
\end{tabular}

glass specimen having the same shape can withstand a local plastic shear strain well in excess of $50 \%$. One obvious application that would capitalize on these qualities is their use as fibres for reinforcement, for example in automobile tyres. Not surprisingly, the mode of deformation exhibited by glassy metals is quite different from that observed in crystalline metals. The inherent weakness of a crystal lies in its ability to be readily dislocated. The virtually un-avoidable presence of dislocations is responsible for the fact that the actual strength of a crystal is seldom more than one-thousandth of the ideal value. It takes surprisingly little force to move a dislocation, and stresses are thus unable to build up to potentially dire levels.

The lack of translational symmetry in the glass precludes a deformation mechanism based on normal dislocation movement, and this is why covalent glasses are always brittle. Remarkably, metallic glasses nevertheless appear to fail by ductile rupture, and the mechanism by which this occurs has understandably aroused interest. In 1972, H.S. Chen, H.J. Leamy, and T.T. Wang observed surface striations on a deformed specimen, suggesting some sort of shear band arrangement. P. Donovan went on to show that if the surface of such a specimen is polished and then etched, the striations reappear, indicating that the sheared zone has been modified by the shearing process. D.E. Polk and David
Turnbull explained this on the basis of alteration of the compositional short-rang $\epsilon$ order, caused by the shear movements. Fractured surfaces of glassy metals display a characteristic vein or ridge pattern, this being reported by Turnbull and F. Spaepen, and independently by C.A. Pampillo and R.C. Reimschuessel. S. Takayama and R. Maddin compared such markings with those formed when two smooth surfaces held together with grease are separated.

The glassy-metal story is still in its infancy but it is already clear that this new type of material is here to stay. The next few years will no doubt bring a mushrooming of new compunds and new applications. Whether or not these developments will be matched by significant advances on the scientific front is perhaps more debatable. One thing at least is assured: glassy metals will continue to be the focus of much attention in university and industrial laboratories.

\title{
The Surfaces of Metals
}

\author{
Derek J. Fabian, Glasgow \\ University of Strathclyde
}

Metal surface physics is fast becoming a field of major significance. Advancements in the physics of surfaces and interfaces generally, especially in connection with materials degradation, have important implications for all of modern technology, from electronic and microprocessor engi-

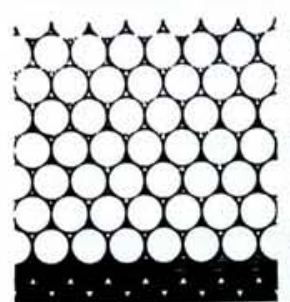

FCC (111)

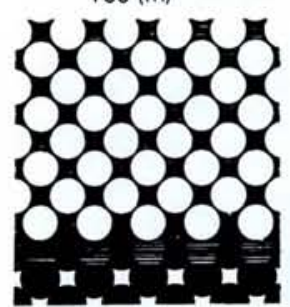

FCC (100)

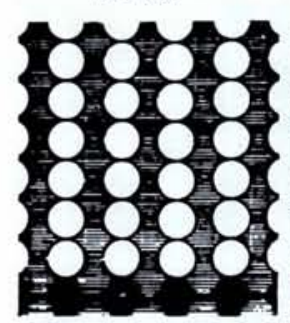

FCC (110)

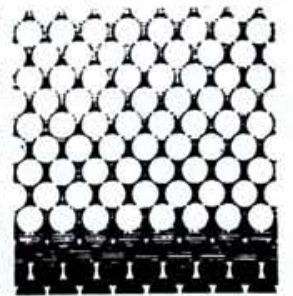
BCC (110)

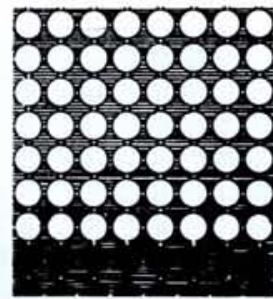

BCC (100)

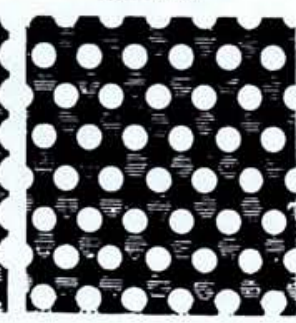

BCC (111)

Fig. 1 -Ideal surface-atom arrangements formed by the high-density planes of face-centred cubic and body-centred cubic metals. neering where semiconductor physicists move constantly to smaller devices, through chemical engineering where chemists and metallurgists continuously seek - for example - an improved understanding of e.g. catalysis, to mechanical engineering where a more thorough knowledge of both the physics and chemistry of processes such as corrosion, oxidation, embrittlement, friction, wear and adhesion is needed, as components become subjected to more extreme conditions. Surface treatments and coatings, and an understanding of thin-film adhesion and surface modification, are already paramount to the further development of many technologies.

\section{Classification of Metal Surfaces}

Surfaces are sometimes sub-divided scientifically into "pure", "clean" and "real" (or "technical"). A pure surface is one in which the metallic lattice is in atomic contact with its adjoining gas (or liquid) phase, and is uncontaminated by adsorbed species. A clean surface might have, for example, a thermodynamically stable thin oxide film, but is free from otherwise adsorbed foreign species. A real or technical surface, more commonly encountered in engineering applications, is one that carries an oxide film plus adsorbed or deposited sometimes embedded - foreign material.

To produce a pure surface, the metal crystal must either be prepared or cleaved in ultra high vacuum, preferably of the order of $10^{-10}$ Torr. A monolayer of contamination, oxide for example, will form on a pure surface in $1 \mathrm{~s}$ when exposed to $10^{-6}$ Torr of residual gas. The resulting metal surface would be classed as "clean". Studies of the more typical metal surfaces found in industrial practice, sometimes

Fig. 2 - Terrace-Ledge-Kink (TLK) model representation of a metallic crystal surface.

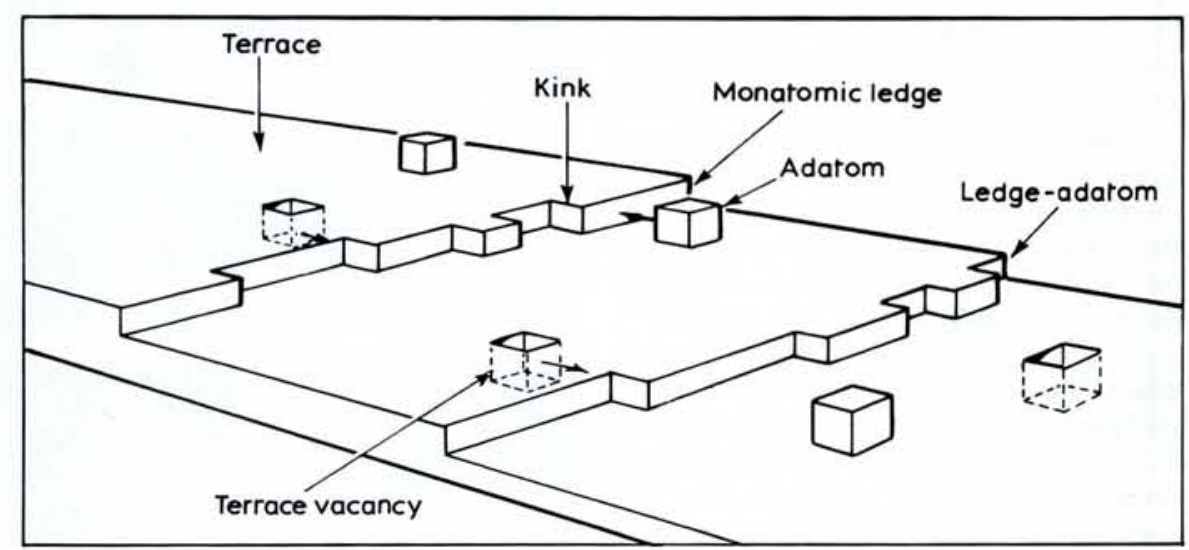

